\title{
Glottic Foreign Body Removal: A Novel Approach
}

\author{
Abhik Sikdar $^{1}$ (D) $\cdot$ Sanjeev Mishra ${ }^{1} \cdot$ Sameer Nivsarkar ${ }^{1} \cdot$ Richa Agrawal $^{1} \cdot$ Shrikant Phatak $^{1}$
}

Received: 27 October 2017 / Accepted: 3 January 2018 / Published online: 16 January 2018

(C) Dr. K C Chaudhuri Foundation 2018

To the Editor: Foreign body in glottis presents as a dire emergency and is mostly fatal before child can access medical care. Securing airway during removal is a major concern.

An 11-mo-old tachypneic male child presented with 1 day history of aspirated broken metal toy. X-ray delineated metallic foil at the level of glottis extending to subglottis. Emergency removal was planned. Airway control was dilemmatic in view of anticipated laryngeal spasm and likely airway bleed during instrumentation. Endotracheal intubation was not feasible. Bag mask ventilation was likely to dislodge the foreign body. Prophylactic tracheostomy would secure the airway but predisposed to the morbidity of tracheal opening in an infant.

We preferred General Anaesthesia with pretracheostomy wherein trachea was exposed and stay sutures passed through anterior tracheal wall for immediate tracheotomy, if required. Anesthetic laryngoscope was introduced and metal piece visualized with camera mounted on transoral $0^{0}$ Hopkins nasal endoscope. This was firmly grasped with bronchoscopy forceps and removed under magnified vision. Check bronchoscopy was normal. There was no trauma or laryngeal spasm. Child was discharged next day with uneventful follow up.

This novel surgical and ventilatory technique allowed us to remove glottic foreign body uneventfully under magnified vision. Only 2 cases of glottic foreign bodies have been published. Kumkum et al. removed foreign body using anaesthetic laryngoscope with Magills forceps [1]. Mundra et al. removed it with ventilating broncoscope without optical instruments [2]. Exposure of anterior tracheal wall without tracheostomy has been documented in a patient undergoing open partial laryngectomy [3]. For glottic foreign bodies such pretracheostomy prevents airway compromise while avoiding the morbidities of a prophylactic tracheostomy in case of successful endolaryngeal ventilation.

\section{Compliance with Ethical Standards}

Conflict of Interest None.

\section{References}

1. Gupta K, Gupta PK. Laryngoscopic removal of unusual metallic foreign body of the subglottic region of the larynx. Anesth Essays Res. 2010;4:106-8.

2. Mundra RK, Agrawal R, Sinha R. Unusual foreign body aspiration in infants below 6 months of age. Indian J Otolaryngol Head Neck Surg. 2014;66:145-8.

3. Britt CJ, Rohrbach MR, McCulloch TM. Tracheal exposure: anticipatory management of the difficult airway. Head Neck. 2016;38: E2446-8.

Abhik Sikdar

abhik_sikdar@yahoo.co.in

1 Department of ENT \& Head and Neck Surgery, Choithram Hospital and Research Centre, Indore, Madhya Pradesh 452014, India 